\title{
Cosmological perturbations in the entangled inflationary universe
}

\author{
Salvador J. Robles-Pérez \\ Estación Ecológica de Biocosmología, Pedro de Alvarado 14, 06411 Medellín, Spain \\ and Instituto de Física Fundamental, CSIC, Serrano 121, 28006 Madrid, Spain
}

(Received 5 November 2017; published 21 March 2018)

\begin{abstract}
In this paper, the model of a multiverse made up of universes that are created in entangled pairs that conserve the total momentum conjugated to the scale factor is presented. For the background spacetime, assumed is a Friedmann-Robertson-Walker metric with a scalar field with mass $m$ minimally coupled to gravity. For the fields that propagate in the entangled spacetimes, the perturbations of the spacetime and the scalar field, whose quantum states become entangled too, are considered. They turn out to be in a quasithermal state, and the corresponding thermodynamical magnitudes are computed. Three observables are expected to be caused by the creation of the universes in entangled pairs: a modification of the Friedmann equation because of the entanglement of the spacetimes, a modification of the effective value of the potential of the scalar field by the backreaction of the perturbation modes, and a modification of the spectrum of fluctuations because the thermal distribution is induced by the entanglement of the partner universes. The later would be a distinctive feature of the creation of universes in entangled pairs.
\end{abstract}

DOI: $10.1103 /$ PhysRevD.97.066018

\section{INTRODUCTION}

One of the most beautiful features of quantum cosmology is the appearance of the classical spacetime and the quantum mechanics of matter fields from the quantum state of the Universe. From the point of view of quantum cosmology, these are emergent features of the semiclassical regime of the wave function of the Universe [1]. This is not very surprising because the wave function of the Universe is obtained by quantizing the Einstein-Hilbert action and the action of the matter fields; so, in a top-down approach, one must recover in the appropriate limit the (semi-) classical behavior of the spacetime and the matter fields.

The wave function of the spacetime and the matter fields, all together, ${ }^{1} \Psi$, can be obtained by solving the Hamiltonian constraint

$$
\hat{H} \Psi=0,
$$

where $\hat{H}$ is the operator form of the Hamiltonian associated to the total action. The Hamiltonian constraint (1) turns out to be a very complicated equation. However, for most of the evolution of the Universe, this is described by a homogeneous and isotropic background with small energy fields propagating therein. In that case, the Hamiltonian constraint (1) can be rewritten as

\footnotetext{
${ }^{1}$ In the context of a single universe $\Psi$ is called the wave function of the universe [2]. However, that name can be misleading in the context of the multiverse, so we here retain the name wave function of the spacetime and matter fields for the wave function that describes the quantum state of the whole spacetime manifold and the matter fields that propagate therein.
}

$$
\left(\hat{H}_{b g}+\hat{H}_{m}\right) \Psi=0
$$

where $H_{b g}$ is the Hamiltonian of the background spacetime and $H_{m}$ contains the matter degrees of freedom. The wave function, $\Psi=\Psi\left(q_{b g}, q_{m}\right)$, where $q_{b g}$ are the degrees of freedom of the background and $q_{m}$ are the matter degrees of freedom, can then be expressed in the semiclassical regime as a linear combination of WKB solutions, i.e., [1],

$$
\Psi\left(q_{b g}, q_{m}\right)=\sum C\left(q_{b g}\right) e^{ \pm \frac{i}{\hbar} S_{0}\left(q_{b g}\right)} \chi\left(q_{b g}, q_{m}\right),
$$

where $C\left(q_{b g}\right)$ is a slow-varying function of the background variables, $S_{0}\left(q_{b g}\right)$ is the action of the background spacetime, and $\chi\left(q_{b g}, q_{m}\right)$ is the wave function of the inhomogeneous degrees of freedom that propagate in the homogeneous and isotropic background. Inserting the semiclassical wave function (3) into the Hamiltonian constraint (2) and solving it in order by order of $\hbar$ in $H_{b g}$, the following is obtained: at zero order, the classical equations of the background spacetime, which provide us with the time variable for the fields that propagate therein, and at first order in $\hbar$ recover the Schrödinger equation of the matter fields with the time variable of the background spacetime provided by the zero-order equations. Spinors and vector fields can also be considered in the semiclassical state (3). Therefore, all the physics we know can, in principle, be derived from a semiclassical state like (3).

Each single addend in (3) can quantum mechanically represent the state of a spacetime background with matter fields propagating therein. The semiclassical state (3) should 
be seen then as the quantum state of a many universe system. However, once the decoherence process has taken place between the branches in $(3)[3,4]$, the customary approach consists of considering one of these branches as the representative of our Universe and disregard the rest of them as being physically redundant or just account them for statistical measures, even though one can still consider other possibilities. For instance, one can look for nonlocal interactions or quantum correlations between the branches in (3). These would ultimately be rooted in a common origin or derived from residual interacting terms of the underlying theory, whether this is one of the string theories or the quantum theory of gravity. In any of these cases, one cannot disregard the rest of the branches of the general state (3).

In particular, in this paper, we are going to study the effects that the creation of universes in entangled pairs may have in the properties of each single universe of the entangled pair. Let us notice that the semiclassical state (3) can be rearranged as

$$
\Psi=\sum C e^{\frac{i}{\hbar} S_{0}} \chi+C^{*} e^{-\frac{i}{\hbar} S_{0}} \chi^{*} .
$$

Each term in the sum (4) can be seen as the wave function of a pair of entangled branches or universes. We show that each branch of the entangled pair has an opposite momentum conjugated to the background variables, and thus, the creation of universes in entangled pairs conserves the total momentum in a parallel way as the creation of particles in entangled pairs conserves the total momentum in a quantum field theory [5]. The creation of universes in entangled pairs would have important consequences in the properties of the matter fields that propagate in their spacetimes because the quantum state of the fields of the two universes become entangled too with a rate of entanglement that depends on the entanglement properties of the parent universes. Then, the effects of the creation of universes in entangled pairs are encoded in the properties of the matter fields that propagate in each single universe, and thus, they might be observed in the properties of our Universe [5].

In this paper, we provide the detailed model of a multiverse made up of universes which are created in entangled pairs. This complements the model presented in Ref. [5], where it is considered a conformally coupled massless scalar field propagating in a homogeneous and isotropic background spacetime. The later provides analytical solutions for the quantum state of the universes and a clear picture of the entanglement processes that may occur in the multiverse. However, we here consider a minimally coupled scalar field with mass $m$, which can mimic more accurately the early stage of our Universe. The paper is outlined as follows. In Sec. II, we obtain the dynamics of the background spacetime and the Schrödinger equation of the matter fields from the semiclassical state of the spacetime and the matter fields. In Sec. III, we show that the most natural way in which the universes can be created is in entangled pairs that conserve the total momentum. In Sec. IV, we impose the boundary condition that the perturbation modes are in the composite vacuum state of the invariant representation that represents a stable vacuum state along the entire evolution of the field. However, in terms of the instantaneously diagonal representation, the invariant vacuum state is full of particle-antiparticle pairs, with the former created in the observer's universe and the antiparticle in the partner one. In Sec. V, we compute the quantum state of the particles in each single universe of the entangled pair and the thermodynamical magnitudes of the field. In Sec. VI, we present three observables that are expected to be caused by the creation of universes in entangled pairs. Finally, in Sec. VII, we summarize and draw some conclusions.

\section{SPACETIME BACKGROUND AND THE QUANTUM MECHANICS OF MATTER FIELDS}

For the background of the model of our Universe, let us consider a homogeneous and isotropic spacetime with the Friedmann-Robertson-Walker (FRW) metric

$$
d s^{2}=-d t^{2}+a^{2}(t) d \Omega_{3}^{2},
$$

where $d \Omega_{3}^{2}$ is the line element on the unit three sphere and a homogeneous and isotropic scalar field, $\phi(t)$, minimally coupled to gravity, with mass $m$ given by

$$
m=V^{\prime \prime}(\phi),
$$

where $V(\phi)$ is the potential of the scalar field. We leave unfixed the functional form of the potential in order to potentially consider different cases including convex $\left(V^{\prime \prime}>0\right)$ as well as concave $\left(V^{\prime \prime}<0\right)$ potentials.

For the fields that propagate in the homogeneous and isotropic background, we consider the small perturbations of the spacetime and the scalar field, i.e., the gravitons and the field particles. These are described by two fields $[6,7]$

$$
\begin{gathered}
h_{i j}(t, \mathbf{x})-a^{2} \Omega_{i j}=a^{2} \sum_{\mathbf{n}} 2 d_{\mathbf{n}}(t) G_{i j}^{\mathbf{n}}(\mathbf{x})+\ldots, \\
\phi(t, \mathbf{x})-\frac{1}{\sqrt{2 \pi}} \phi(t)=\sum_{\mathbf{n}} f_{\mathbf{n}}(t) Q^{\mathbf{n}}(\mathbf{x}),
\end{gathered}
$$

where $Q^{\mathbf{n}}(\mathbf{x})$ are the scalar harmonics on the three sphere and $G_{i j}^{\mathbf{n}}(\mathbf{x})$ the transverse traceless tensor harmonics [6], with $\mathbf{n} \equiv(n, l, m)$. We only focus on the scalar modes of the perturbed field, $f_{\mathbf{n}}$, and the tensor modes of the perturbed spacetime, $d_{\mathbf{n}}$, as representative examples of the matter particles and the gravitons, respectively.

The background degrees of freedom are therefore the scale factor, $a$, and the homogeneous and isotropic part of the scalar field, $\phi$, and the matter degrees of freedom are 
the perturbation modes $f_{\mathbf{n}}$ and $d_{\mathbf{n}}$, denoted generically by $x_{\mathbf{n}}$. The semiclassical wave function of the spacetime and the matter fields, $\Psi\left(a, \phi ; x_{\mathbf{n}}\right)$, is then given by a composite state of the wave function that represents the quantum state of the homogenous and isotropic background, $\Psi_{0}(a, \phi)$, and a wave function that contains the degrees of freedom of the perturbation,

$$
\Psi\left(a, \phi ; x_{\mathbf{n}}\right)=\Psi_{0}(a, \phi) \chi\left(a, \phi ; x_{\mathbf{n}}\right) .
$$

The wave function $\Psi_{0}$ is the solution of the WheelerDeWitt equation of the homogeneous and isotropic background,

$$
\hat{H}_{0} \Psi_{0}=0,
$$

where

$$
\hat{H}_{0}=\frac{1}{2 a}\left(\frac{\partial^{2}}{\partial a^{2}}+\frac{1}{a} \frac{\partial}{\partial a}-\frac{1}{a^{2}} \frac{\partial^{2}}{\partial \phi^{2}}+a^{4} H^{2}(\phi)-a^{2}\right),
$$

with $H^{2}(\phi) \equiv 2 V(\phi)$. In the semiclassical regime, we can consider the WKB solutions of the Wheeler-DeWitt equation (10),

$$
\Psi_{0}^{ \pm}(a, \phi)=C(a, \phi) e^{ \pm \frac{i}{\hbar} S(a, \phi)} .
$$

Inserting the wave function (12) into the Hamiltonian constraint (11), the classical Hamilton-Jacobi equation [7], at zero order in $\hbar$, is satisfied,

$$
-\left(\frac{\partial S}{\partial a}\right)^{2}+\frac{1}{a^{2}}\left(\frac{\partial S}{\partial \phi}\right)^{2}+a^{4} H^{2}(\phi)-a^{2}=0 .
$$

Then, a WKB time parameter $t$ can be defined [7],

$$
\frac{\partial}{\partial t}= \pm \nabla S \cdot \nabla \equiv \pm\left(-\frac{1}{a} \frac{\partial S}{\partial a} \frac{\partial}{\partial a}+\frac{1}{a^{3}} \frac{\partial S}{\partial \phi} \frac{\partial}{\partial \phi}\right)
$$

where $\nabla$ is the gradient of the minisuperspace [8]. In terms of the WKB time (14),

$$
\dot{a}^{2}=\frac{1}{a^{2}}\left(\frac{\partial S}{\partial a}\right)^{2}, \quad \dot{\phi}^{2}=\frac{1}{a^{6}}\left(\frac{\partial S}{\partial \phi}\right)^{2},
$$

and the Hamilton-Jacobi equation (13) turns out to be the Friedmann equation of the background spacetime

$$
\dot{a}^{2}+1-a^{2}\left(\dot{\phi}^{2}+2 V(\phi)\right)=0 .
$$

On the other hand, inserting the wave function (9) into the total Hamiltonian, $H=H_{0}+H_{m}$, where $H_{m}$ is the Hamiltonian of the perturbation modes, obtains the following at first order in $\hbar$ of $H_{0}$ :

$$
\mp i \hbar\left(-\frac{1}{a} \frac{\partial S}{\partial a} \frac{\partial}{\partial a}+\frac{1}{a^{3}} \frac{\partial S}{\partial \phi} \frac{\partial}{\partial \phi}\right) \chi=H_{m} \chi
$$

which is the Schrödinger equation of the matter fields that propagate in the background spacetime (5) provided that the time variable of the Schrödinger equation is the WKB time defined in (14) with the positive sign for the semiclassical wave function $\Psi^{-}$in (12) and the negative sign for $\Psi^{+}$. The wave function (9) can then be written as

$$
\Psi=C e^{+\frac{i}{\hbar} S} \chi_{+}+C^{*} e^{-\frac{i}{\hbar} S} \chi_{-},
$$

with $\chi_{-}=\chi_{+}^{*}$, satisfying the Schrödinger equation (17), i.e.,

$$
i \hbar \frac{\partial}{\partial t_{ \pm}} \chi_{ \pm}=H_{m} \chi_{ \pm}
$$

where $\chi_{ \pm}\left(t, x_{\mathbf{n}}\right) \equiv \chi_{ \pm}\left(a, \phi ; x_{\mathbf{n}}\right)$, evaluated at the background solutions, $a(t)$ and $\phi(t)$ of the Friedmann equation (16). Let us notice that the Friedmann equation is invariant under the time reversal symmetry, $t \leftrightarrow-t$.

If we restrict to small linear perturbations the different modes do not interact [4,9], and $H_{m}$ turns out to be

$$
H_{m}=\sum_{\mathbf{n}} H_{\mathbf{n}},
$$

with

$$
H_{\mathbf{n}}=\frac{1}{2 M} p_{x_{\mathbf{n}}}^{2}+\frac{M \omega_{n}^{2}}{2} x_{\mathbf{n}}^{2}
$$

where $M(t)=a^{3}(t)$ and [7]

$$
\omega_{n}^{2}=\frac{n^{2}-1}{a^{2}},
$$

for the tensorial modes of the spacetime $\left(x_{\mathbf{n}} \equiv d_{\mathbf{n}}\right)$, and

$$
\omega_{n}^{2}=\frac{n^{2}-1}{a^{2}} \pm m^{2}
$$

for the perturbation modes of the scalar field $\left(x_{\mathbf{n}} \equiv f_{\mathbf{n}}\right)$. In (23), the + sign corresponds to the oscillatory phase and the - sign to the inflationary stage of the scalar field $\phi$ [7]. The perturbation modes satisfy then the wave equation of the harmonic oscillator

$$
\ddot{x}_{\mathbf{n}}+\frac{\dot{M}}{M} \dot{x}_{\mathbf{n}}+\omega_{n}^{2} x_{\mathbf{n}}=0 .
$$

The wave function of the perturbation modes can then be written as $[4,7,10]$

$$
\chi=\prod_{\mathbf{n}} \chi_{\mathbf{n}}\left(t, x_{\mathbf{n}}\right),
$$


where the function $\chi_{\mathbf{n}}\left(t, x_{\mathbf{n}}\right)$ is the wave function of a harmonic oscillator with time-dependent mass and frequency, whose general solution can be expanded in the basis of number eigenstates of the invariant representation, $\psi_{N, \mathbf{n}}$, as

$$
\chi_{\mathbf{n}}=\sum_{N} C_{N} \psi_{N, \mathbf{n}}
$$

where $C_{N}$ are constants coefficients, and the wave function of the invariant number state, $\psi_{N, \mathbf{n}}$, is given by $[11,12]$

$$
\begin{aligned}
\psi_{N, n}\left(a, \phi ; x_{\mathbf{n}}\right) & \equiv\left\langle a, \phi ; x_{\mathbf{n}} \mid N_{\mathbf{n}}\right\rangle \\
& =\frac{1}{\sigma^{\frac{1}{2}}} \exp \left\{\frac{i M}{2} \frac{\dot{\sigma}}{\sigma} x_{\mathbf{n}}^{2}\right\} \bar{\psi}_{N}\left(\frac{x_{\mathbf{n}}}{\sigma}, \tau\right),
\end{aligned}
$$

where $\bar{\psi}_{N}(q, \tau)$, with $q \equiv \frac{x_{\mathrm{n}}}{\sigma}$, is the customary wave function of the harmonic oscillator, i.e.,

$$
\bar{\psi}_{N}(q, \tau)=\left(\frac{1}{2^{N} N ! \pi^{\frac{1}{2}}}\right)^{\frac{1}{2}} e^{-i\left(N+\frac{1}{2}\right) \tau} e^{-\frac{q^{2}}{2}} \mathrm{H}_{N}(q),
$$

with $\mathrm{H}_{N}$ being the Hermite polynomial of degree $N$, and $\tau=\tau(t)$ is given by

$$
\tau(t)=\int^{t} \frac{1}{M\left(t^{\prime}\right) \sigma^{2}\left(t^{\prime}\right)} d t^{\prime}
$$

and $\sigma(t)$ is an auxiliary function that satisfies the nonlinear equation $[11,13]$

$$
\ddot{\sigma}+\frac{\dot{M}}{M} \dot{\sigma}+\omega_{n}^{2} \sigma=\frac{1}{M^{2} \sigma^{3}} .
$$

It is worth noticing that a solution of (30) can generally be written as

$$
\sigma=\sqrt{\sigma_{1}^{2}+\sigma_{2}^{2}}
$$

where $\sigma_{1}$ and $\sigma_{2}$ are two independent solutions of (24) satisfying some specific boundary condition. For the boundary condition, one has to realize that in terms of the variable, $z_{\mathbf{n}} \equiv a x_{\mathbf{n}}$, in conformal time $\eta=\int \frac{d t}{a}$, the equation of the harmonic oscillator (24) turns out to be

$$
z_{\mathbf{n}}^{\prime \prime}+n^{2} z_{\mathbf{n}}=0,
$$

in the limit of large modes. In that limit, and in terms of the variable $z_{\mathbf{n}}$, the wave function of the modes should be then the customary wave function of the harmonic oscillator with unit mass and constant frequency $n$. This is accomplished if we impose that

$$
\sigma \rightarrow \frac{1}{\sqrt{M \omega_{n}}} \approx \frac{1}{a \sqrt{n}}, \quad \dot{\sigma} \rightarrow 0,
$$

in the limit of large modes for all time. Thus, the computation of the wave function of the perturbation modes essentially reduces to the computation of the solutions of (30) that satisfy the boundary condition (33). Their quantum state, however, will depend on the boundary condition that we impose on the state of the field, and this, in turn, will depend on the boundary condition imposed on the state of the universes. In particular, it is shown that in the context of the creation of universes in entangled pairs it depends on the rate of entanglement between the universes.

\section{CREATION OF UNIVERSES IN ENTANGLED PAIRS}

During the early stage of the Universe, the potential of the field can be considered approximately constant for the tiny amount of time for which the universe is exponentially expanding. In that case, the wave function of the Universe, $\Psi$, can be easily expanded in partial waves

$$
\Psi(a, \phi)=\int \frac{d K}{\sqrt{2 \pi}} e^{i K \phi} \Psi_{K}(a) \chi_{K}\left(a ; x_{\mathbf{n}}\right),
$$

where the amplitude, $\Psi_{K}(a) \equiv \Psi_{K}\left(a, \phi_{0}\right)$, satisfies the Wheeler-DeWitt-like equation

$$
\frac{\partial^{2} \Psi_{K}}{\partial a^{2}}+\frac{1}{a} \frac{\partial \Psi_{K}}{\partial a}+\Omega_{K}^{2}(a) \Psi_{K}(a)=0
$$

with

$$
\Omega_{K}=\sqrt{H^{2} a^{4}-a^{2}+\frac{K^{2}}{a^{2}}},
$$

where $H^{2} \equiv V\left(\phi_{0}\right)$, evaluated at some initial value $\phi_{0}$. The wave function of the modes, $\chi_{K}\left(a ; x_{\mathbf{n}}\right) \equiv \chi_{K}\left(a, \phi_{0} ; x_{\mathbf{n}}\right)$, satisfies then the Schrödinger equation (19) with the time variable $t$ of the corresponding background spacetime.

However, the structure of the wave function $\Psi$ in (4) and (18) suggests that it can be seen as a field to be quantized following a formalism that parallels that of a quantum field theory, which is called the third quantization formalism $[14,15]$. Following the parallelism, the constants $C$ and $C^{*}$ in (4) and (18) are promoted to quantum operators that eventually turn out to be the creation and annihilation operators of universes, in a many-particle description of the quantum field $\hat{\Psi}$. Each component $\Psi^{ \pm}$in (4) and (18) represents a universe with an opposite value of the momentum conjugated to the configuration variables, i.e., the momentum conjugated to the scale factor and the momentum conjugated to the scalar field, whose signs are essentially determined by the signs of the exponents of the WKB solutions involved in (4) and (18), 


$$
\begin{aligned}
& \hat{p}_{a} \Psi^{ \pm}=-i \hbar \frac{\partial \Psi^{ \pm}}{\partial a} \approx \pm \frac{\partial S}{\partial a}, \\
& \hat{p}_{\phi} \Psi^{ \pm}=-i \hbar \frac{\partial \Psi^{ \pm}}{\partial \phi} \approx \pm \frac{\partial S}{\partial \phi} .
\end{aligned}
$$

In that context, and holding on the parallelism with the quantum field formalism, the most natural way in which the universes should be created is in pairs with opposite values of their momenta that would conserve the total momentum. ${ }^{2}$ In that case, the perturbation modes of the scalar field and the gravitons of the spacetime of each universe propagate in their corresponding background spacetimes separately but in a quantum state that can be correlated with the quantum state of the perturbations of the partner universe. Their quantum states may become entangled too.

The creation of universes in entangled pairs can be easily visualized as the Lorentzian continuation of a double Euclidean instanton $[17,18]$. However, it can be considered a more general feature in quantum cosmology. In the particular case of (34), where the scalar field is assumed to be constant, the action $S$ of the WKB solutions can be seen as a function of the scale factor alone. Then, one can formally proceed as it is usually done in a quantum field theory and express the wave function (34) in terms of two linearly independent solutions of the Wheeler-DeWitt equation (35). For these, let us take the WKB solutions, given by

$$
\Psi_{K}^{ \pm} \approx \frac{1}{\sqrt{a \Omega_{K}(a)}} e^{ \pm i \int \Omega_{K}(a) d a},
$$

which are normalized according to $\Psi_{K}^{*} \partial_{a} \Psi_{K}-\Psi_{K} \partial_{a} \Psi_{K}^{*}=$ $\pm \frac{2 i}{a}$. Then, (34) can be written as

$\Psi(a, \phi)=\int \frac{d K}{\sqrt{2 \pi}}\left(e^{i K \phi} \Psi_{K}^{+} \chi_{K} b_{K}+e^{-i K \phi} \Psi_{K}^{-} \chi_{K}^{*} c_{K}^{*}\right)$,

where $b_{K}$ and $c_{K}^{*}$ are two constants that are promoted to quantum operators, $b_{K} \rightarrow \hat{b}_{K}$ and $c_{K}^{*} \rightarrow \hat{c}_{K}^{\dagger}$, in the third quantization formalism $[14,15]$. The wave function (39) describes then the quantum state of pairs of entangled universes that are created with opposite momenta, given by $[5,17]$

$$
\left\langle\hat{p}_{a}\right\rangle \approx \pm \Omega_{K}
$$

at leading order. The pair of newborn universes conserve thus the total momentum conjugated to the scale factor. The process parallels the creation of particles in entangled pairs with opposite momenta that conserve the total momentum in a quantum field theory described in an isotropic background

\footnotetext{
${ }^{2}$ For more details, the reader can see Refs. [5,16-18] and references therein.
}

spacetime. Therefore, in the context of the multiverse, the creation of universes in entangled pairs seems to be the most natural way in which the universes can be created [5].

The momentum conjugated to the scale factor, however, depends on the expansion rate of the universe. The two WKB branches in (38) correspond then to an expanding and a contracting branch of the spacetime in terms of the time variable $t$. This can be seen by noticing that in the semiclassical regime the expected value of the momentum conjugated to the scale factor (40) is highly peaked around the classical value, $p_{a}^{c} \equiv-a \dot{a}$ [see also (15)]. Thus,

$$
-a \dot{a}=\mp \Omega_{K},
$$

which is nothing more than the Friedmann equation associated to the Wheeler-DeWitt equation (35),

$$
\frac{\dot{a}^{2}}{a^{2}}=\frac{\Omega_{K}^{2}}{a^{4}} \Rightarrow \dot{a}= \pm \frac{\Omega_{K}}{a},
$$

for the two branches $\Psi^{ \pm}$. Nevertheless, the WKB time variable $t$ is not the time experienced by the internal observers in their particle physics experiments. Let us notice that the value of the momentum conjugated to the scale factor determines the value of the time variable in each single universe. Thus, according to (41), the time variable in one of the universes of the entangled pair is defined by

$$
\frac{\partial}{\partial t_{I}}=\frac{\Omega_{K}}{a} \frac{\partial}{\partial a},
$$

and the time variable in the partner universe by

$$
\frac{\partial}{\partial t_{I I}}=-\frac{\Omega_{K}}{a} \frac{\partial}{\partial a} .
$$

The time variables of the entangled universes are related by an antipodal-like symmetry [19], $t_{I}=-t_{I I}$, and the two branches turn out to be expanding branches in terms of the time variables $t_{I}$ and $t_{I I}$. These are the time variables experienced by the internal observers in their particle physics experiments $[5,20]$, which are governed by the Schrödinger-like equation

$$
\mp i \frac{\Omega_{K}}{a} \frac{\partial}{\partial a} \chi=H_{m} \chi .
$$

This is the usual Schrödinger equation with the time variable of each single universe of the entangled pair provided that the time variables in the two universes are taken to be $t_{I}$ and $t_{I I}$ defined by (43) and (44), respectively. Therefore, $t_{I}$ and $t_{I I}$ are the time variables measured by their actual clocks, and the two entangled universes become expanding universes from the point of view of the internal observers. 
The wave function (39) can now be written as

$$
\Psi=\int \frac{d K}{\sqrt{2 \pi}}\left(e^{i K \phi} \Psi_{K}^{+} \chi_{K}^{I} \hat{b}_{K}+e^{-i K \phi} \Psi_{K}^{-} \chi_{K}^{I I} \hat{c}_{K}^{\dagger}\right),
$$

where $\chi_{K}^{I}$ and $\chi_{K}^{I I}$, with $\chi_{K}^{I I}=\left(\chi_{K}^{I}\right)^{*}$, are the wave functions of the perturbation modes in each single universe of the entangled pair. They satisfy the Schrödinger equation (19) with $t_{ \pm}$being replaced by $t_{I}$ or by $t_{I I}$ and $\chi_{ \pm}$by $\chi_{K}^{I}$ and $\chi_{K}^{I I}$, respectively. In the case that the matter content of the universe is represented by a complex scalar field, then matter would be always created in the observer's universe and antimatter in the partner universe, restoring thus the matter-antimatter symmetry in the pair of entangled universes [20].

\section{QUANTUM STATE OF THE PERTURBATION MODES}

In the context of the creation of universes in entangled pairs, the matter particles and the gravitons of each universe propagate in the background spacetime of their corresponding universe and follow the Schrödinger equation (19) with the time variable of the corresponding background spacetime. The wave function $\chi_{K}^{I, I I}$ defines the quantization of the perturbation modes in the Schrödinger picture, where $\hat{x}_{\mathbf{n}}$ and $\hat{p}_{x_{\mathbf{n}}}$ are time-independent operators that act on the time-dependent wave function $|\chi(t)\rangle$. In the configuration space of the amplitude of the perturbation modes, the Hilbert space is spanned by the basis, $\left\{\prod_{\mathbf{n}}\left|x_{\mathbf{n}}\right\rangle\right\}$, where the vectors $\left|x_{\mathbf{n}}\right\rangle$ are the eigenvectors of the amplitude operators $\hat{x}_{\mathbf{n}}$. The general quantum state of the perturbations is then given by

$$
\left|\chi_{K}(t)\right\rangle=\int \prod_{\mathbf{n}} d x_{\mathbf{n}} \chi\left(x_{\mathbf{n}}, t\right) \prod_{\mathbf{n}}\left|x_{\mathbf{n}}\right\rangle .
$$

In the case of small perturbations, for which the different modes do not interact among them and $\chi\left(x_{\mathbf{n}}, t\right)$ can be written as in (25), it can be written that

$$
\left|\chi_{K}(t)\right\rangle=\prod_{\mathbf{n}} \int d x_{\mathbf{n}} \chi_{\mathbf{n}}\left(x_{\mathbf{n}}, t\right)\left|x_{\mathbf{n}}\right\rangle,
$$

where $\chi_{\mathbf{n}}\left(x_{\mathbf{n}}, t\right)=\left\langle x_{\mathbf{n}} \mid \chi(t)\right\rangle$.

However, the development of the corresponding quantum field theory seems to be more useful. This can be done, as usual, by considering the wave equation of the fields that represent the inhomogeneous degrees of freedom, given by (7) and (8), the general solution of the harmonic oscillator (24) with $\omega_{n}$ being given by (22) in the case of the perturbation modes of the scalar field, and by (23) in the case of the gravitons of the gravitational field. It can then be written that

$$
x_{\mathbf{n}}(t)=v_{n}^{*}(t) a_{\mathbf{n}}^{-}+v_{n}(t) b_{-\mathbf{n}}^{+},
$$

where $v_{n}(t)$ and $v_{n}^{*}(t)$ are two linearly independent solutions of the harmonic oscillator (24), and $a_{\mathbf{n}}^{-}$and $b_{\mathbf{n}}^{+}$ are two constants satisfying, $b_{\mathbf{n}}^{+}=\left(a_{\mathbf{n}}^{-}\right)^{*}$. Then, the development of the quantum field theory of the perturbation modes in the two entangled universes follows by promoting the constants, $a_{\mathbf{n}}^{-}$and $b_{\mathbf{n}}^{+}$, to quantum operators, $a_{\mathbf{n}}^{-} \rightarrow \hat{a}_{\mathbf{n}}$ and $b_{\mathbf{n}}^{+} \rightarrow \hat{b}_{\mathbf{n}}^{\dagger}$, satisfying the customary commutation relations. In the picture of a pair of universes created in an entangled state, the symmetry of the composite state (46) suggests that matter is created in the observer's universe and antimatter in the partner universe [20]. Let us notice that for the observer of the partner universe it is the other way around. Therefore, $\hat{a}_{\mathbf{n}}^{\dagger}$ and $\hat{a}_{\mathbf{n}}$ are the creation and annihilation operators of matter in one universe, and $\hat{b}_{\mathbf{n}}^{\dagger}$ and $\hat{b}_{\mathbf{n}}$ are the creation and annihilation of matter in the other universe, satisfying both the corresponding commutation relations. In the case of a real field, the particles are their own antiparticles. However, the creation and annihilation operators of modes of the two universes commute among them because to the Euclidean gap between the universes $[18,20]$ we retain the different names $\hat{a}_{\mathbf{n}}$ and $\hat{b}_{\mathbf{n}}$. Then, the inhomogeneous part of the mode decomposition given in (7) and (8) can be written quantum mechanically as

$$
x(\mathbf{x}, t)=\sum_{\mathbf{n}} v_{n}^{*}(t) F_{\mathbf{n}}(\mathbf{x}) \hat{a}_{\mathbf{n}}+v_{n}(t) F_{\mathbf{n}}^{*} \hat{b}_{\mathbf{n}}^{\dagger},
$$

where $F_{n}(\mathbf{x})$ are the scalar harmonics on the three sphere, $Q^{\mathbf{n}}(\mathbf{x})$, in the case of the perturbation modes of the scalar field, and $F_{\mathbf{n}}$ are the transverse traceless tensor harmonics, $G_{i j}^{\mathbf{n}}(\mathbf{x})$, in the case of the gravitons.

We can define the vacuum state of the particles in the two universes as $|0\rangle_{I}$ and $|0\rangle_{I I}$ as

$$
|0\rangle_{I, I I}=\prod_{\mathbf{n}}\left|0_{\mathbf{n}}\right\rangle_{I, I I}
$$

where the states $\left|0_{\mathbf{n}}\right\rangle$ are the states annihilated by $\hat{a}_{\mathbf{n}}$ in universe $I$ and by $\hat{b}_{\mathbf{n}}$ in universe $I I$. On the other hand, with the operators $\hat{a}_{\mathbf{n}}^{\dagger}$ and $\hat{b}_{\mathbf{n}}^{\dagger}$, we can build the customary orthonormal bases for the corresponding Hilbert spaces,

$$
\prod_{\mathbf{n}}\left|N_{\mathbf{n}}\right\rangle_{I}=\prod_{\mathbf{n}} \frac{1}{\sqrt{N_{\mathbf{n}} !}}\left(\hat{a}_{n}^{\dagger}\right)^{N_{\mathbf{n}}}\left|0_{\mathbf{n}}\right\rangle_{I}
$$

and a similar one with $\hat{b}_{n}^{\dagger}$ instead of $\hat{a}_{n}^{\dagger}$ for universe $I I$. An arbitrary quantum state of the perturbations can then be written as a linear combination of the excited states,

$$
|\chi\rangle_{I, I I}=\sum_{\mathbf{n}} \sum_{N_{\mathbf{n}}} C_{N_{\mathbf{n}}, N_{\mathbf{n}^{\prime}} \ldots} \prod_{\mathbf{n}}\left|N_{\mathbf{n}}\right\rangle_{I, I I}
$$

with constants coefficients, $C_{N_{\mathbf{n}}, N_{\mathbf{n}^{\prime}} \ldots}$. 
The quantum state of the perturbation modes are now determined by the boundary condition imposed on the states of the perturbation modes. This, in turn, depends on the boundary condition imposed on the state of the hosting universes. In the case of a pair of entangled universes, we impose that the perturbation modes are in the composite vacuum state of the invariant representation [21]. The invariant representation has the great advantage that once the field is in a number state of the invariant representation it remains in the same state along the entire evolution of the field. In particular, once the field is in the vacuum state of the invariant representation, it remains in the same vacuum state along the entire evolution of the field. It seems to be then an appropriate boundary condition for the perturbation modes of the pair of entangled universes. The invariant representation of the harmonic oscillator (24) can be written as $[13,15]$

$$
\begin{gathered}
\hat{a}_{\mathbf{n}}=\sqrt{\frac{1}{2}}\left(\frac{1}{\sigma} x_{\mathbf{n}}+i\left(\sigma p_{x_{\mathbf{n}}}-M \dot{\sigma} x_{\mathbf{n}}\right)\right), \\
\hat{b}_{-\mathbf{n}}^{\dagger}=\sqrt{\frac{1}{2}\left(\frac{1}{\sigma} x_{\mathbf{n}}-i\left(\sigma p_{x_{\mathbf{n}}}-M \dot{\sigma} x_{\mathbf{n}}\right)\right) .}
\end{gathered}
$$

The perturbation modes are then in the vacuum state of the invariant representation given by

$$
|0\rangle=\left|0_{a} 0_{b}\right\rangle=\left|0_{a}\right\rangle_{I}\left|0_{b}\right\rangle_{I I} .
$$

However, the particles of the scalar field and the gravitons measured by the internal observers of the universe are not described by the number states of the invariant representations [(54), (55)]. They are instead described by the number states of the instantaneous diagonal representation of the Hamiltonian of the harmonic oscillator (21), which defines the instantaneous vacuum state at each moment of time. In terms of the diagonal representation, the amplitude of the perturbations and their conjugate momenta can be written as

$$
\begin{aligned}
x_{\mathbf{n}} & =\frac{1}{\sqrt{2 M \omega_{n}}}\left(c_{\mathbf{n}}+d_{-\mathbf{n}}^{\dagger}\right), \\
p_{x_{\mathbf{n}}} & =-i \sqrt{\frac{M \omega_{n}}{2}}\left(c_{\mathbf{n}}-d_{-\mathbf{n}}^{\dagger}\right) .
\end{aligned}
$$

The invariant representation [(54), (55)] can be related to the diagonal representation [(57), (58)] through the Bogolyubov transformation,

$$
\begin{gathered}
a_{\mathbf{n}}=\mu(t) c_{\mathbf{n}}-\nu^{*}(t) d_{-\mathbf{n}}^{\dagger}, \\
b_{-\mathbf{n}}=\mu(t) d_{-\mathbf{n}}-\nu^{*}(t) c_{\mathbf{n}}^{\dagger},
\end{gathered}
$$

where $\mu \equiv \mu_{n}$ and $\nu \equiv \nu_{n}$ are given by

$$
\begin{aligned}
& \mu(t)=\frac{1}{2}\left(\sigma \sqrt{M \omega_{n}}+\frac{1}{\sigma \sqrt{M \omega_{n}}}-i \dot{\sigma} \sqrt{\frac{M}{\omega_{n}}}\right), \\
& \nu(t)=\frac{1}{2}\left(\sigma \sqrt{M \omega_{n}}-\frac{1}{\sigma \sqrt{M \omega_{n}}}-i \dot{\sigma} \sqrt{\frac{M}{\omega_{n}}}\right),
\end{aligned}
$$

with $|\mu|^{2}-|\nu|^{2}=1$ for all time.

Let us now compute the quantum state of the perturbation modes in one single universe of the entangled pair. From (56), the composite state of the perturbation modes in the two entangled universes can be written in the density matrix formalism as

$$
\rho=\left|0_{a} 0_{b}\right\rangle\left\langle 0_{a} 0_{b}\right| .
$$

Using the Bogolyubov transformation [(59), (60)], the vacuum state of the invariant representation, $\left|0_{a} 0_{b}\right\rangle$, can be written as [22]

$$
\left|0_{a} 0_{b}\right\rangle=\prod_{\mathbf{n}} \frac{1}{|\mu|}\left(\sum_{N=0}^{\infty}\left(\frac{\nu}{\mu}\right)^{N}\left|N_{c, \mathbf{n}} N_{d,-\mathbf{n}}\right\rangle\right),
$$

where

$$
\left|N_{c, \mathbf{n}}\right\rangle=\frac{\left(c_{\mathbf{n}}^{\dagger}\right)^{N}}{\sqrt{N !}}\left|0_{c, \mathbf{n}}\right\rangle, \quad\left|N_{d,-\mathbf{n}}\right\rangle=\frac{\left(d_{-\mathbf{n}}^{\dagger}\right)^{N}}{\sqrt{N !}}\left|0_{d,-\mathbf{n}}\right\rangle
$$

are the number states of the diagonal representation [(57), (58)]. It means that the vacuum state of the invariant representation is full of particles of the scalar field and gravitons of the gravitational field. The number and properties of the particles depend on the parameters $\mu$ and $\nu$, and thus, they depend on the rate of entanglement between the universes. The effects of the entanglement between the two universes can thus be indirectly observed because they are encoded in the observable state of the perturbation modes. These effects have no classical analogue, so they should entail distinguishable features of the interuniversal entanglement and of the whole multiverse proposal too.

\section{QUANTUM THERMODYNAMICS OF THE PERTURBATION MODES}

Let us consider the quantum state of the particles and gravitons in just one single universe of the entangled pair. The reduced density matrix that represents the quantum state of the particles in one single universe alone can be obtained by tracing out from the density matrix (63) the state of the particles in the partner universe. It yields $[15,23,24]$

$$
\rho_{c}=\operatorname{Tr}_{d} \rho=\prod_{\mathbf{n}} \frac{1}{Z_{n}} \sum_{N} e^{-\frac{1}{T_{n}}\left(N+\frac{1}{2}\right)}\left|N_{c, \mathbf{n}}\right\rangle\left\langle N_{c, \mathbf{n}}\right|,
$$


where $Z_{n}^{-1}=2 \sinh \frac{1}{2 T_{n}}$, and

$$
T_{n} \equiv T_{n}(t)=\frac{1}{\ln \frac{|\mu(t)|^{2}}{|\nu(t)|^{2}}}=\frac{1}{\ln \left(1+|\nu(t)|^{-2}\right)} .
$$

The density matrix $\rho_{c}$ represents a quasithermal state whose thermal properties depend on the rate of entanglement between the universes. In particular, the specific temperature of entanglement (67) is a measure of the entanglement [23] between the field particles and the gravitons of the two entangled universes. The largest modes of the particles and gravitons do not feel the effect of the entanglement because for large modes $\nu \rightarrow 0$ and $T \rightarrow 0$, so these modes are in the vacuum states. However, for the shorter modes, the effects of the interuniversal entanglement may be significant.

A caveat should be made before going on. For appearance, the state (66) is not properly a thermal state in the sense of classical thermodynamics, for at least two reasons. First, it is not derived from thermalizing interactions between microscopic subsystems. Rather, it is obtained just from the process of tracing out the degrees of freedom of the perturbation modes of the partner universe from the composite vacuum state (63). It is therefore a measure of the entanglement between the two universes. Second, the quantum distribution (66) cannot represent a classical thermal state unless the temperature, $T_{n}$, would be the same for all modes. ${ }^{3}$ We are not dealing therefore with classical thermodynamics but with quantum thermodynamics [25-27], which is expected to be a generalization of the former [28], although the relationship between both formalisms is not clear yet. ${ }^{4}$ One would expect that in some semiclassical limit the temperature of entanglement associated to each mode would converge to a constant value for all modes, i.e., $T_{n} \rightarrow T$, for which the properties of thermal equilibrium and ergodicity of the classical formulation of thermodynamics should be recovered. However, as we already said, this process is not clear yet. In the present work, we assume that $T_{n}$ is a measure of entanglement between the modes of the two universes without ascribing to it any specific thermal property, at least in the classical sense.

Therefore, the modes are not really thermalized until the temperature $T_{n}$ becomes the same for all modes, even though one can define, for each mode, the thermodynamical magnitudes of entanglement associated to the quantum state (66). They are given by $[16,26,27]$

$$
E(a)=\operatorname{Tr}\left(\hat{\rho}_{c}(a) \hat{H}(a)\right),
$$

\footnotetext{
${ }^{3}$ Let us also notice that $T_{n}$ in (67) is not exactly the temperature but the specific temperature, which is the temperature divided by the frequency $\omega_{n}$.

${ }^{4}$ The relationship between the thermodynamics of entanglement and the classical formulation of thermodynamics is a subject of intense research (see, for instance, Refs. [25,28-32]).
}

$$
\begin{aligned}
& Q(a)=\int^{a} \operatorname{Tr}\left(\frac{d \hat{\rho}_{c}\left(a^{\prime}\right)}{d a^{\prime}} \hat{H}\left(a^{\prime}\right)\right) d a^{\prime}, \\
& W(a)=\int^{a} \operatorname{Tr}\left(\hat{\rho}_{c}\left(a^{\prime}\right) \frac{d \hat{H}\left(a^{\prime}\right)}{d a^{\prime}}\right) d a^{\prime},
\end{aligned}
$$

where $\operatorname{Tr}(\hat{O})$ stands for the trace of the operator $\hat{O}, H$ is the Hamiltonian of the harmonic oscillator (21), and the sums and integrals have to be performed within the Hubble horizon [see Eq. (91)]. For the density matrix $\rho_{c}$ in (66), it yields $[5,16]$

$$
\begin{gathered}
E_{n}(t)=\frac{\omega_{n}}{2} \operatorname{cotanh} \frac{1}{2 T_{n}}=\omega_{n}\left(N_{n}+\frac{1}{2}\right), \\
Q_{n}(t)=\frac{\omega_{n}}{2} \operatorname{cotanh} \frac{1}{2 T_{n}}-\omega_{n} T_{n} \ln \sinh \frac{1}{2 T_{n}}, \\
W_{n}(t)=\omega_{k} T_{n} \ln \sinh \frac{1}{2 T_{n}},
\end{gathered}
$$

where $N_{n} \equiv|\nu|^{2}$. The first principle of thermodynamics, $d E_{n}(t)=\delta Q_{n}(t)+\delta W_{n}(t)$, is satisfied for all modes $\mathbf{n}$ individually, and the energy densities that correspond to $E_{n}$, $Q_{n}$, and $W_{n}$, are given by

$$
\varepsilon_{n}=\frac{E_{n}}{V}, \quad q_{n}=\frac{Q_{n}}{V}, \quad w_{n}=\frac{W_{n}}{V},
$$

with $V=a^{3}(t)$ being the volume of the space. The entropy of entanglement [16,33] can also be easily obtained from the von Neumann formula

$$
S\left(\rho_{c}\right)=-\operatorname{Tr}\left(\rho_{c} \ln \rho_{c}\right),
$$

with $\rho_{c}$ given by (66). It yields [34]

$$
S_{\mathrm{ent}}(a)=|\mu|^{2} \ln |\mu|^{2}-|\nu|^{2} \ln |\nu|^{2},
$$

from which it can be checked that the second principle of thermodynamics is also satisfied [16].

It is worth noticing that the energy of the vacuum state of the invariant representation is the same as the energy of the quantum state (66) of the diagonal representation. The former is given by

$$
E_{0}^{I}={ }_{I}\langle 0|H| 0\rangle_{I}
$$

It yields [11]

$$
E_{0}^{I}=\frac{\omega_{n}}{4}\left(\sigma^{2} M \omega_{n}+\frac{1}{\sigma^{2} M \omega_{n}}+\frac{M \dot{\sigma}^{2}}{\omega_{n}}\right) .
$$

On the other hand, the energy of the quantum state (66) in the diagonal representation is given by (71) with, $N_{n}=|\nu|^{2}$, which can be also written as 


$$
E_{t h}^{D}=\frac{\omega_{n}}{2}\left(|\mu|^{2}+|\nu|^{2}\right) .
$$

By using the values of $\mu$ and $\nu$ given in (61) and (62), it can be checked that (78) and (79) yield the same value. The energy is therefore conserved, as it was expected, and the quantum state $\rho_{c}$ in (66) entails just a redistribution of the modes with the same total energy.

\section{OBSERVABLE IMPRINTS}

Three potentially observable effects are expected to be caused by the creation of universes in entangled pairs. First, the boundary condition imposed on the state of the universes may modify the effective value of their Friedmann equation, given initially by (42). Let us notice that the Wheeler-DeWitt equation (35) can be formally considered as the generalized equation of a harmonic oscillator with time-dependent mass, $\mathcal{M}(a)=a$, and frequency, $\Omega_{K}(a)$, given by (36), with the scale factor formally playing the role of the time variable. The quantization of that generalized harmonic oscillator is the basis of the so-called third quantization formalism. In that context, a similar argument to that applied to the perturbation modes can be given for the states of the wave function $\Psi_{K}$. We can then impose that the quantum state of the homogeneous and isotropic background should be the vacuum state of the invariant representation associated to the generalized harmonic oscillator (35). The vacuum state of the invariant representation, which is a stable ground state along the entire evolution in the minisuperspace, turns out to be full of entangled pairs of number states of the diagonal representation [5]. The expected value of the generalized Hamiltonian $\mathcal{H}$ of the harmonic oscillator (35) is given, similarly to (78), by

$$
\begin{aligned}
\langle 0|\mathcal{H}| 0\rangle & =\frac{\Omega_{K}}{4}\left(R^{2} \mathcal{M} \Omega_{K}+\frac{1}{R^{2} \mathcal{M} \Omega_{K}}+\frac{\mathcal{M}}{\Omega_{K}}\left(\frac{d R}{d a}\right)^{2}\right) \\
& \equiv \frac{\tilde{\Omega}_{K}}{2} .
\end{aligned}
$$

Then, the evolution of the entangled universes is effectively determined by a modified Friedmann equation given now, instead of by (42), by

$$
\frac{d a}{d t}= \pm \frac{\tilde{\Omega}_{K}}{a} .
$$

In the adiabatic limit, for a large value of the scale factor,

$$
R \approx \frac{1}{\sqrt{\mathcal{M} \Omega_{K}}} .
$$

Then,

$$
\tilde{\Omega}_{K} \approx \Omega_{K}\left(1+\frac{1}{8 \Omega_{K}^{2}}\left(\frac{d}{d a} \log \left(\mathcal{M} \Omega_{K}\right)\right)^{2}\right) .
$$

With the values $\mathcal{M}=a$ and $\Omega_{K} \approx H(\phi) a^{2}$, the following is obtained:

$$
\tilde{\Omega}_{K} \approx H a^{2}+\frac{9}{8 H a^{4}} .
$$

The modified Friedmann equation (81) yields

$$
a(t)=a_{0}\left(e^{6 H \Delta t}-1\right)^{\frac{1}{6}} .
$$

At late times, the scale factor (85) evolves in an exponential way. However, the entanglement between the universes produce a preinflationary stage in the evolution of the universe that might leave observable consequences in the properties of the cosmic microwave background (CMB). Let us notice that although inflation is supposed to wash out most of the imprints of a preinflationary stage of the universe, some preinflationary scenarios would entail a suppression of the lowest modes of the power spectrum of the CMB that is compatible with the astronomical data $[35,36]$, provided that inflation does not last for too long, i.e., in the so-called justenough inflation scenarios. In that case, the preinflationary evolution of the universe caused by the entanglement with a partner universe might leave observable consequences, which is currently under investigation [37].

Another modification of the Friedmann equation that is expected to leave observable imprints in the properties of our universe is the backreaction of the perturbation modes, given by the energy density associated to the energy (78) [or (79)]. In the case of an exactly flat DeSitter expansion, the value of $\sigma$ that satisfies the boundary condition (33) is given by (31) with $\sigma_{1}$ and $\sigma_{2}$ given by

$$
\begin{aligned}
\sigma_{1} & =\sqrt{\frac{\pi}{2 H}} a^{-\frac{3}{2}} \mathcal{J}_{q}\left(\frac{n}{H a}\right), \\
\sigma_{2} & =\sqrt{\frac{\pi}{2 H}} a^{-\frac{3}{2}} \mathcal{Y}_{q}\left(\frac{n}{H a}\right),
\end{aligned}
$$

where $\mathcal{J}_{q}(x)$ and $\mathcal{Y}_{q}(x)$ are the Bessel functions of first and second kind and order $q=\sqrt{\frac{9}{4}-\frac{m^{2}}{H^{2}}}$. For the case, $m \ll H$ $\left(q \approx \frac{3}{2}\right), \sigma$ turns out to be

$$
\sigma^{2} \approx \frac{H^{2} a^{2}+n^{2}}{a^{2} n^{3}}
$$

and the energy (78) yields

$$
E_{n}=\frac{H x}{2}\left(1+\frac{1}{2}\left(1+\frac{m^{2}}{H^{2}}\right) x^{-2}+2 \frac{m^{2}}{H^{2}} x^{-4}\right),
$$

with

$$
x \equiv \frac{n}{H a}=\frac{n_{\mathrm{ph}}}{H} \sim \frac{H^{-1}}{L_{\mathrm{ph}}},
$$


where $L_{\mathrm{ph}}$ is the physical wave length and $H^{-1}$ is the distance to the Hubble horizon. The problem now is that the energy of the backreaction (89) turns out to be divergent when it is summed over all modes. A cutoff has to be imposed. Following Refs. [38,39], the energy of the modes can be integrated from the value $n=a b$, where $b$ is the SUSY breaking scale of the subjacent landscape, to the value $n=a H$, disregarding thus the superhorizon modes. Then, the following is obtained:

$$
\begin{aligned}
\varepsilon & =\frac{1}{V} \int_{a b}^{a H} d n n^{2} E_{n} \\
& =\frac{H^{4}}{8}\left\{1-\frac{m^{2}}{H^{2}} \log \frac{b^{2}}{H^{2}}+\left(1+\frac{m^{2}}{H^{2}}\right)\left(1-\frac{b^{2}}{H^{2}}\right)\right\},
\end{aligned}
$$

where terms of higher order have been disregarded. The energy shift (91) can be seen as a correction to the effective value of the potential of the scalar field, an effect that has also been studied in Refs. [38,39] (see, also, Refs. [40-42]), where it is concluded that a similar correction to that given in (91) would entail a suppression of the power spectrum at large angular scales, running of the spectral index, and a suppression of the $\sigma_{8}$ parameter [38,39].

Finally, the third effect that is expected to leave observable imprints in the properties of the CMB is the spectrum of fluctuation of the quantum state (66) caused by the entanglement between the universes. The fluctuations of the perturbation modes can be obtained from

$$
\delta \phi_{\mathbf{n}}=\frac{n^{\frac{3}{2}}}{2 \pi} \Delta \phi_{n}
$$

where

$$
\left(\Delta \phi_{n}\right)^{2}=\left\langle\left|\phi_{n}\right|^{2}\right\rangle-\left|\left\langle\phi_{n}\right\rangle\right|^{2}
$$

In the vacuum state of the invariant representation [11], $\left|\left\langle\phi_{n}\right\rangle\right|=0$ and

$$
\left\langle\left|\phi_{n}\right|^{2}\right\rangle=\frac{\sigma^{2}}{2} .
$$

In the case of a DeSitter spacetime, $\sigma_{1}$ and $\sigma_{2}$ in (86) and (87) are the real and imaginary parts of the Bunch-Davies vacuum, so $\sigma$ is essentially the modulus of the BunchDavies modes. Thus, the fluctuations of the invariant vacuum, given by (92) with (94), turn out to yield the customary expression [22]

$$
\delta \phi_{\mathbf{n}}=\frac{H}{\sqrt{8 \pi}} x^{\frac{3}{2}}\left(\mathcal{J}_{q}^{2}(x)+\mathcal{Y}_{q}^{2}(x)\right)^{\frac{1}{2}} .
$$

However, the creation of universes in entangled pairs induces the perturbation modes to be in the quantum state (66) of the diagonal representation, and then,

$$
\begin{aligned}
\left\langle\left|\phi_{n}\right|^{2}\right\rangle & =\frac{1}{M \omega_{n}}\left(|\nu|^{2}+\frac{1}{2}\right) \\
& =\frac{1}{4 M \omega_{n}}\left(\sigma^{2} M \omega_{n}+\frac{1}{\sigma^{2} M \omega_{n}}+\frac{M \dot{\sigma}^{2}}{\omega_{n}}\right) .
\end{aligned}
$$

With the value of $\sigma$ given by (88), it turns out that

$$
\frac{\delta \phi_{\mathbf{n}}^{\text {th }}}{\delta \phi_{\mathbf{n}}^{I}}=\sqrt{\frac{1}{2}\left(1+\frac{x^{2}}{\left(1+x^{2}\right)\left(1+\frac{m^{2}}{H^{2} x^{2}}\right)}\right)} .
$$

The large modes $(x \gg 1)$ are in the vacuum state, and then, $\delta \phi_{\mathbf{n}}^{\text {th }} \approx \delta \phi_{\mathbf{n}}^{I}$. However, the departure could be relevant for the horizon modes $x \sim 1$. It is worth noticing that this is not an effect derived from a preinflationary evolution of the universe, so it is not expected to be washed out by inflation. It is an effect derived from a different choice of the initial state of the perturbation modes, which would be stem from the existence of a partner universe whose quantum mechanical state is entangled with ours. Although the departure of the quantum state (66) from the vacuum state is expected to be small because the entanglement between the universes, and thus the temperature of entanglement (67), is expected to be small too, the residual effect that it would entail might still produce observable imprints. Even though the possibility of finding this effect is currently quite small, the analysis of the present work is still important because it would represent a distinctive effect with no classical analogue in the context of an isolated universe, and therefore, it would be a distinguishable effect of the whole multiverse proposal.

\section{SUMMARY AND CONCLUSIONS}

We have presented a detailed model of a multiverse made up of pairs of universes whose quantum mechanical states are entangled. The existence of the multiverse, although bizarre at first sight, is something that has been implicitly considered from the very beginning of quantum cosmology. Each semiclassical branch of the general solution of the Wheeler-DeWitt equation represents a spacetime background with matter fields propagating therein; i.e., it represents a different realization of the universe. The customary approach has generally consisted of considering one of these branches as the representative of our universe and disregarding the rest of them because it seems meaningless to physically consider external elements to the universes. However, we have shown that quantum correlations and other nonlocal interactions may exist between the states of the universes and that they may leave observable imprints in the properties of a universe like ours. In particular, we have shown that the creation of universes in entangled pairs correlate the quantum states of the matter fields that propagate in their respective spacetimes. 
We have shown as well that the most natural way in which the universes can be created is in entangled pairs that conserve the momentum conjugated to the scale factor, in a parallel way as particles are created in entangled pairs that conserve the total momentum in a quantum field theory. The momentum conjugated to the scale factor, however, depends on the expansion rate of the universes. Thus, the opposite values of the momentum in the pair of universes is related to the opposite expansion rates of the universes in terms of a common time variable. Nevertheless, the time experienced by the internal observers in their particle physics experiments, i.e., the time measured by actual clocks, are related by an antipodal like symmetry, $t_{I}=-t_{I I}$. Then, from the point of view of the internal observers, the universes are both expanding universes.

The quantum states of the particles of the matter fields and the gravitons of the spacetime that propagate in the two entangled universes become entangled too. The most appropriate boundary condition seems to be that the fields are in the composite vacuum state of the invariant representation. This is a stable representation of the vacuum state along the entire evolution of the fields. However, in terms of the instantaneous diagonal representation of the corresponding Hamiltonian, which would represent the state of the particles measure by internal observers, the quantum state of the field turns out to be given by a quasithermal state whose thermodynamical magnitudes of entanglement depend on the rate of entanglement between the universes. Thus, the interuniversal properties of entanglement may be encoded in the quantum state of the matter fields that propagate in our universe.
We expect three observable effects caused by the creation of universes in entangled pairs. The first one would be caused by the entanglement of the background spacetimes of the universes. It would modify the effective value of the Friedmann equation by introducing a preinflationary stage in the evolution of the universe that might leave observable imprints in the properties of the CMB provided that inflation does not last for too long. A second effect would be caused by the backreaction of the inhomogeneous degrees of freedom. It would entail a modification of the effective value of the potential of the scalar field that would have a direct consequence in the properties of the inflationary expansion and, thus, in the observed properties of the early universe. Finally, the spectrum of fluctuations of the perturbation modes for the thermal state induced by the entanglement of the partner universes is significantly different from the one expected from an unentangled universe. This is then a distinctive feature of the creation of the universes in entangled pairs that has no analogue in the context of an isolated universe. It is therefore a distinguishable feature of the whole multiverse proposal.

We have shown therefore that the multiverse is a testable proposal. The process that may happen in the multiverse would eventually leave their imprints in the observable properties of the single universes, and thus, they become testable. The door is now open for the study of a wide variety of new cosmic phenomena. Let us notice that these effects are expected to be residual effects of the underlying theory, whether this is one of the string theories of the quantum theory of gravity. Thus, they may help us to test these most fundamental theories.
[1] J. B. Hartle, in Quantum Cosmology and Baby Universes, edited by S. Coleman, J. B. Hartle, T. Piran, and S. Weinberg (World Scientific, London, UK, 1990), Vol. 7, p. 67.

[2] J. B. Hartle and S. W. Hawking, Wave function of the universe, Phys. Rev. D 28, 2960 (1983).

[3] J. J. Halliwell, Decoherence in quantum cosmology, Phys. Rev. D 39, 2912 (1989).

[4] C. Kiefer, Decoherence in quantum electrodynamics and quantum gravity, Phys. Rev. D 46, 1658 (1992).

[5] S. Robles-Pérez, Quantum cosmology of a conformal multiverse, Phys. Rev. D 96, 063511 (2017).

[6] J. J. Halliwell and S. W. Hawking, Origin of structure in the universe, Phys. Rev. D 31, 1777 (1985).

[7] C. Kiefer, Continuous measurement of mini-superspace variables by higher multipoles, Classical Quantum Gravity 4, 1369 (1987).

[8] J. J. Halliwell, in Quantum Cosmology and Baby Universes, edited by S. Coleman, J. B. Hartle, T. Piran, and S. Weinberg (World Scientific, London, UK, 1990), Vol. 7, p. 159.
[9] J. J. Halliwell, Correlations in the wave function of the universe, Phys. Rev. D 36, 3626 (1987).

[10] L. P. Grishchuk and Y. V. Sidorov, Squeezed quantum states of relic gravitons and primordial density fluctuations, Phys. Rev. D 42, 3413 (1990).

[11] P. G. L. Leach, Harmonic oscillator with variable mass, J. Phys. A 16, 3261 (1983).

[12] S. J. Robles-Pérez (to be published).

[13] H. R. Lewis and W. B. Riesenfeld, An exact quantum theory of the time-dependent harmonic oscillator and of a charged particle in a time-dependent electromagnetic field, J. Math. Phys. (N.Y.) 10, 1458 (1969).

[14] A. Strominger, in Quantum Cosmology and Baby Universes, edited by S. Coleman, J. B. Hartle, T. Piran, and S. Weinberg (World Scientific, London, UK, 1990), Vol. 7, p. 272.

[15] S. Robles-Pérez and P. F. González-Díaz, Quantum state of the multiverse, Phys. Rev. D 81, 083529 (2010).

[16] S. Robles-Pérez and P. F. González-Díaz, Quantum entanglement in the multiverse, J. Exp. Theor. Phys. 118, 34 (2014). 
[17] I. Garay and S. Robles-Pérez, Effects of a scalar field on the thermodynamics of interuniversal entanglement, Int. J. Mod. Phys. D 23, 1450043 (2014).

[18] S. J. Robles-Pérez, Creation of entangled universes avoids the big bang singularity, J. Gravity 2014, 382675 (2014).

[19] A. Linde, Inflation and quantum cosmology, Phys. Scr. T T36, 30 (1991).

[20] S. J. Robles-Pérez, Restoration of matter-antimatter symmetry in the multiverse, arXiv:1706.06304.

[21] S. Robles-Pérez, Invariant vacuum, Phys. Lett. B 774, 608 (2017).

[22] V.F. Mukhanov and S. Winitzki, Quantum Effects in Gravity (Cambridge University Press, Cambridge, England, 2007).

[23] S. Robles-Pérez, A. Balcerzak, M. P. Dąbrowski, and M. Kramer, Inter-universal entanglement in a cyclic multiverse, Phys. Rev. D 95, 083505 (2017).

[24] S. J. Robles-Pérez, Observational consequences of an interacting multiverse, Universe 3, 49 (2017).

[25] M. B. Plenio and V. Vedral, Teleportation, entanglement and thermodynamics in the quantum world, Contemp. Phys. 39, 431 (1998).

[26] R. Alicki, M. Horodecki, P. Horodecki, and R. Horodecki, Thermodynamics of quantum informational systemsHamiltonian description, Open Syst. Inf. Dyn. 11, 205 (2004).

[27] J. Gemmer et al., Quantum Thermodynamics (SpringerVerlag, Berlin, Germany, 2009).

[28] M. Horodecki, Quantum entanglement: Reversible path to thermodynamics, Nat. Phys. 4, 833 (2008).

[29] F. G. S. L. Brandao and M. B. Plenio, Entanglement theory and the second law of thermodynamics, Nat. Phys. 4, 873 (2008).

[30] M. Hossein-Partovi, Entanglement versus stosszahlansatz: Disappearance of the thermodynamic arrow in a highcorrelation environment, Phys. Rev. E 77, 021110 (2008).
[31] D. Jennings and T. Rudolph, Entanglement and the thermodynamic arrow of time, Phys. Rev. E 81, 061130 (2010).

[32] P. Ćwikliński, M. Studziński, M. Horodecki, and J. Oppenheim, Limitations on the Evolution of Quantum Coherences: Towards Fully Quantum Second Laws of Thermodynamics, Phys. Rev. Lett. 115, 210403 (2015).

[33] R. Horodecki, P. Horodecki, M. Horodecki, and K. Horodecki, Quantum entanglement, Rev. Mod. Phys. 81, 865 (2009).

[34] S. Robles-Pérez, Entanglement arrow of time in the multiverse, Open Access Library J. 1, 1 (2012).

[35] F. Scardigli, C. Gruber, and P. Chen, Black hole remnants in the early universe, Phys. Rev. D 83, 063507 (2011).

[36] M. Bouhmadi-Lopez, P. Chen, and Y. Liu, Cosmological imprints of a generalized chaplygin gas model for the early universe, Phys. Rev. D 84, 023505 (2011).

[37] M. Bouhmadi-López, M. Krämer, J. Morais, and S. RoblesPérez, Pre-inflation from the multiverse: Can it solve the quadrupole problem in the cosmic microwave background?, arXiv:1711.05138.

[38] R. Holman, L. Mersini-Houghton, and T. Takahashi, Cosmological avatars of the landscape. I. Bracketing the supersymmetry breaking scale, Phys. Rev. D 77, 063510 (2008).

[39] R. Holman, L. Mersini-Houghton, and T. Takahashi, Cosmological avatars of the landscape ii, Phys. Rev. D 77, 063511 (2008).

[40] L. Mersini-Houghton, Predictions of the quantum landscape multiverse, Classical Quantum Gravity 34, 047001 (2017).

[41] E. Di Valentino and L. Mersini-Houghton, Testing predictions of the quantum landscape multiverse 1: The starobinsky inflationary potential, J. Cosmol. Astropart. Phys. 03 (2017) 002.

[42] E. Di Valentino and L. Mersini-Houghton, Testing predictions of the quantum landscape multiverse 2: The exponential inflationary potential, J. Cosmol. Astropart. Phys. 03 (2017) 020. 